\title{
Torsion theca lutein cyst in association with invasive mole presenting as acute abdomen: a rare case
}

\section{Radhamani S., Sushma V. Dev*, Akhila M.V.}

Department of Obstetrics and Gynaecology, Mysore Medical College and Research Institute, Mysore, Karnataka, India

Received: 14 June 2015

Accepted: 10 July 2015

\author{
*Correspondence: \\ Dr. Sushma V. Dev, \\ E-mail: docsushmavdev@gmail.com
}

Copyright: (C) the author(s), publisher and licensee Medip Academy. This is an open-access article distributed under the terms of the Creative Commons Attribution Non-Commercial License, which permits unrestricted non-commercial use, distribution, and reproduction in any medium, provided the original work is properly cited.

\begin{abstract}
Gestational trophoblastic neoplasias (GTN) are rare tumours that constitute less than $1 \%$ of all gynaecological malignancies. Invasive mole is a distinct subgroup of GTN, which if not diagnosed and treated early, can result in serious complications like uterine perforation and haemoperitoneum. We present a rare case of torsion theca lutein cyst in association with invasive mole of the uterus, which developed following the evacuation of a molar pregnancy with features of continued irregular vaginal bleeding, persistently high $\beta$ Hcg levels along with acute abdomen.
\end{abstract}

Keywords: Beta-human chorionic gonadotropin, Gestational trophoblastic neoplasia (GTN), Invasive mole, Theca lutein cyst, Torsion, PSTT

\section{INTRODUCTION}

Gestational Trophoblastic Disease (GTD) is a term used for a group of pregnancy related disorders arising from abnormal placental trophoblast cells. It encompasses two pre-malignant conditions: partial and complete hydatidiform moles and the malignant gestational trophoblastic neoplasias (GTN). GTNs are classified histologically into three distinct subgroups: choriocarcinoma destruens (invasive mole), choriocarcinoma (CC) and the very rare placental site trophoblastic tumour (PSTT). ${ }^{1}$ Invasive moles are responsible for most cases of localized GTN. It follows approximately $10-15 \%$ of complete hydatidiform moles and $0.5 \%$ of patients with partial mole. ${ }^{2}$ Invasive mole are characterized by presence of whole chorionic villi that accompany excessive trophoblastic overgrowth and invasion. This tissue penetrates deep into the myometrium sometimes involving the peritoneum or vaginal vault. Such moles are locally invasive but generally lack the pronounced tendency to develop widespread metastases typical of choriocarcinoma.

\section{CASE REPORT}

A 24 year old lady, gravida 3 para 2 living 1 was referred to our tertiary care institute for evaluation of recurrent episodes of bleeding per vagina following post molar evacuation. There was history of molar pregnancy for which she had undergone suction evacuation twice in private hospital two months back. Her initial and post evacuation $\beta$ HCG level were $30,500 \mathrm{miu} / \mathrm{ml}$ and 3019 $\mathrm{miu} / \mathrm{ml}$ respectively. Histo-pathological examination revealed it to be complete mole.

At the time of admission, vitals were stable with normal per abdominal examination. Per vaginal examination showed minimal bleeding. Bimanual examination revealed uterus enlarged to 8 weeks gestational age size and bilateral palpable adnexal cyst. Pelvic ultrasound with Colour Doppler imaging reported as enlarged uterus with a hypervascular mass in the myometrium invading the myometrium, suspicious of invasive mole with grossly enlarged theca lutein cysts. Magnetic resonance imaging (MRI) confirmed invasive mole with focal myometrial invasion into posterior wall and right lateral 
wall. Also it showed bilateral large ovarian theca lutein cysts measuring $11 * 8 \mathrm{~cm}$ and $8 * 6 \mathrm{cms}$ (Figure 1).
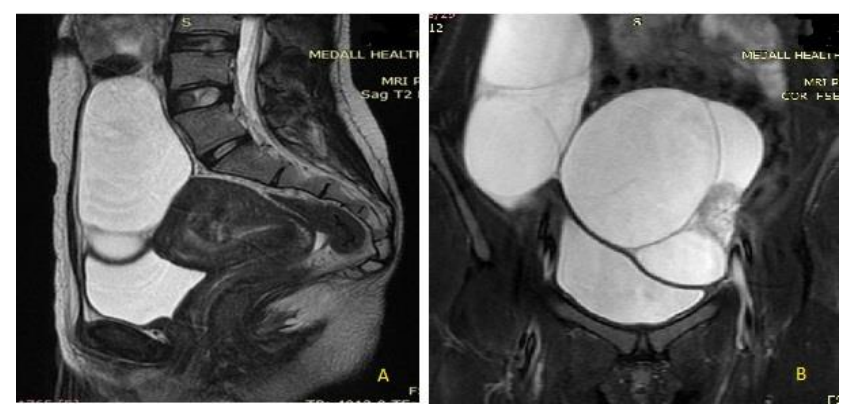

Figure 1: MRI images: A) sagittal T2 image showing invasive mole with myometrial invasion into posterior wall, B) coronal MRI shows bilateral large theca lutein cysts.

As the followup $\beta \mathrm{HCG}$ was $815 \mathrm{miu} / \mathrm{ml}$ which showed a decreasing trend oncologist advised regular $\beta \mathrm{HCG}$ monitoring on outpatient basis and to decide on chemotherapy after looking into subsequent $\beta \mathrm{HCG}$ values. She came back a week later to our emergency with acute pain abdomen having deranged vitals and severe pallor. Per abdomen examination revealed a 24 wk. size pelvic mass with tenderness, guarding and rigidity. Bleeding per vaginum was minimal. Uterine perforation was suspected and patient was taken up for emergency laparotomy. Intraoperative findings revealed torsion of left ovarian theca lutein cyst which was haemorrhagic, grossly enlarged to $15^{*} 17 \mathrm{~cm}$ and an enlarged right theca lutein cyst. Uterus was bulky with serosa intact (Figure 2).

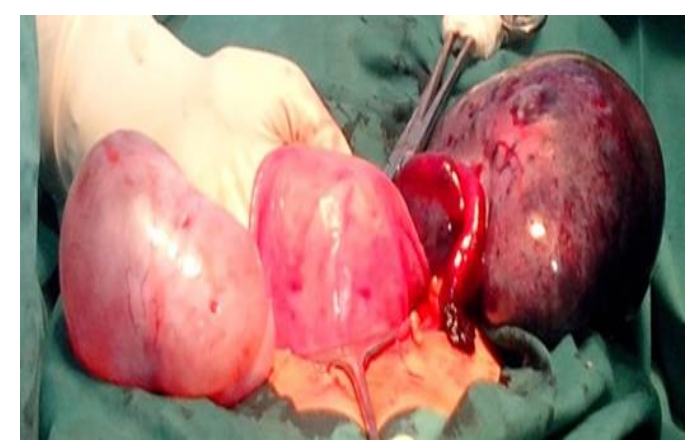

Figure 2: Intraoperative image showing haemorrhagic left ovarian theca lutein cyst with torsion.

Total abdominal hysterectomy with left ovariotomy was done. Right ovary was preserved. Cut section of the uterus showed a molar tissue occupying whole of uterine fundus with invasion of myometrium at posterior wall (Figure 3). Histopathology report of the uterus confirmed invasive mole with no vascular emboli (Figure 4) and the cyst showing features of torsion with transmural infarction and haemorrhage. Her post-operative period was uneventful with beta HCG value of $20 \mathrm{miu} / \mathrm{ml}$.

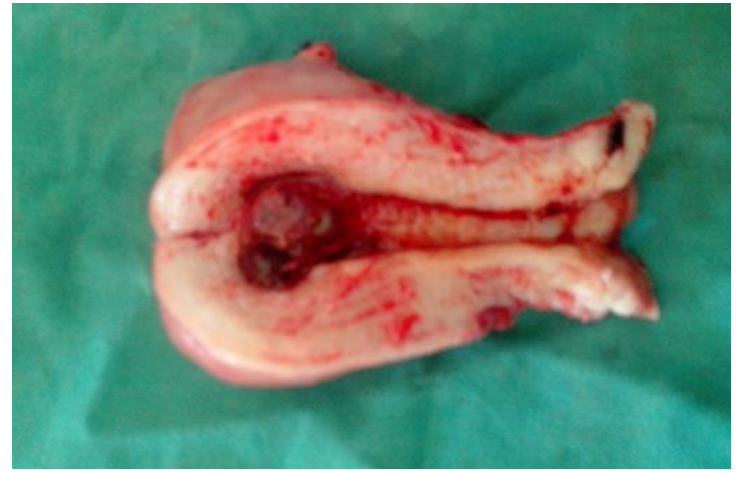

Figure 3: Cut-section of uterus showing invasive mole in posterior wall.

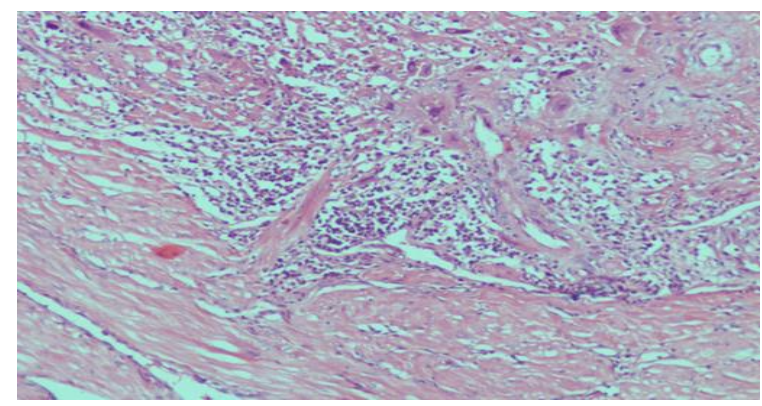

Figure 4: Histopathology of invasive mole showing myometrial invasion of villi.

\section{DISCUSSION}

GTN are rare and constitute less than $1 \%$ of all gynaecological malignancies. Originating in placental tissue, they are characterized by a distinct tumour marker (beta HCG) and have varying tendencies towards local invasion and distant metastasis. ${ }^{3}$ Approximately $8 \%$ of patients with complete moles will develop a malignant tumour after evacuation. ${ }^{3-5}$ Therefore $\beta H C G$ should be monitored after evacuation, which if persistently elevated, should raise the suspicion of GTN.

An invasive mole is a form of GTN that occurs most commonly after the evacuation of hydatidiform mole usually within six months. It is characterized by the presence of edematous chorionic villi with trophoblastic proliferation that invades into the myometrium of the uterus or to adjacent structures like the vagina, vulva, broad ligament, and can also invade into the uterine vessels. ${ }^{5,6}$ Invasive mole appears histologically benign but the clinical course of invasive mole is malignant. Clinically it presents as vaginal bleeding, an enlarged uterus and high urinary or serum $\beta \mathrm{HCG}$ level. If left untreated invasive mole can result in heavy bleeding, uterine rupture or other symptoms from local invasion. , $^{4,8}$ Direct vascular metastasis rarely occurs, the most common site reported is the lung. Myometrial invasion is difficult to document on pelvic ultrasound and also in uterine curetting's unless there is a sufficient myometrium to demonstrate the invasion. Colour Doppler imaging and MRI are novel tools to demonstrate 
myometrial invasion. It is important to distinguish between invasive mole and choriocarcinoma, as the former has a more favourable outcome.

PSTTs are predominately composed of intermediate cytotrophoblast cells arising from the placental implantation site. ${ }^{9}$ PSTTs can result from any type of antecedent pregnancy and are usually locally aggressive, producing myometrial invasion. Histologic identification of PSTT or radiographic identification of bulky disease in conjunction with a low HCG level should suggest the possibility of this lesion. ${ }^{9}$ Hysterectomy should be employed as primary therapy for cases of nonmetastatic PSTTs. In contradistinction to invasive moles and gestational CC, PSTT is usually resistant to conventional chemotherapy used in the treatment of GTD.

Doppler velocimetric findings in patients with invasive mole, PSTT and CC are similar in that they all may exhibit low-impedance arterial flow and high velocity. Bilateral enlarged ovaries due to theca lutein cysts may also be seen. ${ }^{3}$ It is possible to distinguish between these entities, using a combination of both biochemical findings and ultrasound appearances.

In $25 \%-60 \%$ of the cases of hydatidiform mole, the ovaries are seen to contain multiple theca lutein cysts, resulting from ovarian hyperstimulation due to high circulating levels of $\beta \mathrm{HCG}^{10,11,12}$ These cysts are multiloculated and often bilateral. Ovarian enlargement correlates with marked elevation of serum $\beta \mathrm{HCG}$ levels greater than $100,000 \mathrm{mIU} / \mathrm{ml}$. Although theca lutein cysts are usually detected before molar evacuation, they often develop within first week after evacuation. ${ }^{10,12}$ The mean time for disappearance of these cysts is approximately 8 weeks. Theca lutein cysts are associated with an increased incidence of post molar trophoblastic tumour; in particular, Montez and colleagues reported a $75 \%$ incidence of post molar sequelae among women with bilateral theca lutein cysts. ${ }^{12}$ Approximately $30 \%$ of theca lutein cysts develop secondary enlargement in response to rising $\beta \mathrm{HCG}$ levels associated with post molar sequelae. ${ }^{12}$ In nature, it is a benign condition, the appropriate management is conservative, but surgical management requiring oophorectomy is definitely indicated when theca lutein cysts present with uncommon serious complications as ovarian torsion or rupture and bleeding from the cyst. ${ }^{13}$

In our case, the patient had elevated $\beta H C G$ and myometrial invasion supportive of GTN. Marginal high $\beta \mathrm{HCG}$ was not discriminative of either invasive mole or PSTT. The acute presentation of the case with torsion made us to lean more towards PSTT with risk of impending perforation hence TAH with unilateral oophorectomy was done. Post-surgical histopathological report confirmed it to be invasive mole.

Even in the presence of disseminated disease, most of the cases are amenable to treatment with almost $100 \%$ survival. Surgical intervention plays an important role in managing patients of trophoblastic disease presenting with acute abdomen and emergency situations like suspected uterine perforation, torsion of theca lutein cyst, severe vaginal bleeding. ${ }^{13}$ Although the development of effective chemotherapy has resulted in improved survival of the patients with GTN, hysterectomy remains an important adjunct in the treatment of the selected subset of patients. ${ }^{14}$ Surgical therapy (hysterectomy) performed coincident with the institution of the chemotherapy has shown that it significantly reduces the duration of hospitalization and amount of chemotherapy used to achieve remission. In this case, we did not give any chemotherapy because there was no evidence of metastasis and due to the declining values of $\beta$ hCG.

Even though theca lutein cysts are common association of GTN, torsion is a rare occurrence seen in less than $2 \%$ of cases and only few cases being reported. Our case depicts rare combination of torsion of theca lutein cyst and invasive mole.

\section{CONCLUSION}

Through this case report we emphasise the importance of regular serial assay of $\beta \mathrm{HCG}$ and clinical assessment of progress in cases of molar pregnancy following evacuation. Acute life threatening situation may be an initial presenting clinical scenario of persistent mole and GTN. High index of suspicion is important for early diagnosis and treatment. Surgical management always remains as an important adjunct in selected subset of patients and in emergency situations.

\section{Funding: No funding sources \\ Conflict of interest: None declared \\ Ethical approval: Not required}

\section{REFERENCES}

1. Ngan HY, Bender H, Benedet JL, Jones H, Montruccoli GC, Pecorelli S. FIGO Committee on Gynecologic Oncology. Gestational trophoblastic neoplasia, FIGO 2000 staging and classification. Int J Gynaecol Obstet. 2003;83 Suppl 1:175-77

2. Hammond CB. Gestational trophoblastic neoplasms. In: Scott JR, DiSaia PJ, and Spellacy WN (Eds). Danforth's Obstetrics and Gynecology, 8th ed. Philadelphia: Lippincott Williams \& Wilkins 1999:927-937.

3. Moodley M, Tunkyi K, Moodley J. Gestational trophoblastic syndrome: an audit of 112 patients. A South African experience. Int J Gynecol Cancer 2003;13:234-9.

4. Kavitha Nair, Hanaa Al-Khawari. Invasive mole of the uterus - a rare case diagnosed by ultrasound: a case report. Med Ultrason 2014;16(2):175-7.

5. Soper JT, Mutch DG, Schink JC. American College of Obstetricians and Gynecologists. Diagnosis and treatment of gestational trophoblastic disease: ACOG 
Practice Bulletin No.53. Gynecol Oncol 2004;93:575-85.

6. Maeda K, Kurjak A, Varga G, Honemeyer U. Trophoblastic diseases. DSJUOG. 2012;6:27-42.

7. Amandeep K, Shashi G, Vikram M, Rajni G. Invasive mole presenting as acute abdomen. J\&K India 2011;13:35-6.

8. Mackenzie F, Mathers A, Kennedy J. Invasive hydatidiform mole presenting as an acute primary haemoperitoneum. $\mathrm{Br}$ J Obstet Gynecol 1993; 100:953-54

9. Mazur MT, Kurman RJ: Choriocarcinoma and placental site tumor. In Szulman AE, Buchsbaum HJ (Eds): Gestational Trophoblastic Disease. New York, Springer- Verlag, 1987;45-68.

10. Morrow CP, Kletzky OA, DiSaia PJ et al: Clinical and laboratory correlates of molar pregnancy and trophoblastic disease. Am J Obstet Gynecol 1977;128:424-9.
11. Curry SL, Hammond CG, Tyrey L et al: Hydatidiform mole: Diagnosis, management and long-term follow up of 347 patients. Obstet Gynecol 1975;45:1-8.

12. Montz FJ, Schlaerth JB, Morrow CP: The natural history of theca lutein cysts. Obstet Gynecol 1988;72:247-51.

13. Xiang Y, Yang X, Zhang L. Evaluation of emergency surgery in gestational trophoblastic tumours. Zhongguo Yi Xue Ke Xue Yuan Xue Bao. 1997;19(5):369-72.

14. Xiang Y, Yang X, Du J. The role of hysterectomy in the therapy of gestational trophoblastic tumor. Zhonghua Zhong Liu Za Zhi. 1999;21:139-41.

Cite this article as: Radhamani S, Dev SV, Akhila MV. Torsion theca lutein cyst in association with invasive mole presenting as acute abdomen: a rare case. Int J Reprod Contracept Obstet Gynecol 2015;4:1237-40. 